\title{
Development and Assessment of VR/AR Solution for Verification During the Construction Process
}

\author{
Kostiantyn Protchenko ${ }^{1, *}$, Piotr Dąbrowski ${ }^{1}$, and Andrzej Garbacz ${ }^{1}$ \\ ${ }^{1}$ Warsaw University of Technology, Civil Engineering Department, Lecha Kaczyńskiego St., 16, \\ 00-637 Warsaw, Poland
}

\begin{abstract}
It is common occurrence that on-site workers misunderstand various detailing aspects of the provided documentation. Some of them can be unclear and should be discussed again due to construction related processes. In order to deal with this issue the application for mobile devices was proposed. The developed application allows to visualize twodimensional print projects with the help of VR/AR (Virtual Reality/Augmented Reality) technology. The paper is focused on the assessment of the possibility to use the VR/AR technology during the process of construction. The feasibility of the solution is tested on the basis of a three-dimensional model created with the software operating in accordance with Building Information Modeling (BIM) technology. The developed mobile VR/AR solution was enhanced with basic functionality to verify structural members as well as entire structural system. Moreover, it allows to communicate in a simple manner, which can lead to the improving of the effectiveness and preventing the risk of construction errors.
\end{abstract}

\section{Introduction}

The development and implementation of Building Information Modeling (BIM) technology in various phases of the project is one of the current trends in construction industry. Designing with the instruments, which operate in accordance with BIM technology (BIM software) is associated with an increased workload during concept and design phases [1], however, the associated automation gives the opportunity to obtain additional benefits mainly due to obtaining an accurate three-dimensional model filled with necessary information [2].

BIM-compliant model enables efficient collaboration between all project partners, improved documentation workflow, and improved-quality project results, via enhanced performance analysis and multi-disciplinary planning and coordination [3,4 and 5]. Additionally, it allows to represent and interpret information about it outside the BIM environment. Use of Virtual Reality/Augmented Reality (VR/AR), as an instruments for Information and Communication Technology (ICT), can be considered as one of

\footnotetext{
* Corresponding author: k.protchenko@il.pw.edu.pl
} 
accomplishments that can be possible with BIM model [6]. The VR/AR technology provide with the possibility of additional verifying, coordination and communication, and can be used during the construction phase, even under construction conditions.

This paper describes the VR/AR solution for mobile devices, which enables to display structural members in three-dimensional view. The conditions that are necessary for the correct operation of the developed application have been analyzed, and the potential use was assessed basing on realistic condition of construction process.

\section{The Prerequisites for Developed Application}

The developed mobile application was created using previously prepared BIM model, which was based on the monolithic building with reinforced concrete structure. The BIM model of the building was developed using BIM software, which fulfills requirements that were assumed for the purpose of developing the application using the augmented reality. The first prerequisite is that the BIM software should support an export in the appropriate file formats, such as: *.3ds, *.fbx, *.skp etc. that allows to reproduce it in VR/AR modes with the help of Unity environment [7, 8].

The authors decided to use Unity environment due to the built-in numerous facilities for operating in a three-dimensional environment, including the embedded graphics engine for the Android platform. Additionally, it contains a tool for reproducing three-dimensional models with the help of augmented reality, which is called - Vuforia [9].

The initial task was to obtain the same representation of elements and details on the screen of the mobile device as that one on the computer screen. By exporting the BIM model to the *.3ds format, the geometry remains unchanged from the original. However, it is worth mentioning that *.3ds format serves for transferring objects' geometry only. Figure 1a shows the animation view of the reinforced concrete column made in BIM software and Figure $1 \mathrm{~b}$ shows its representation in the developed application.

a)

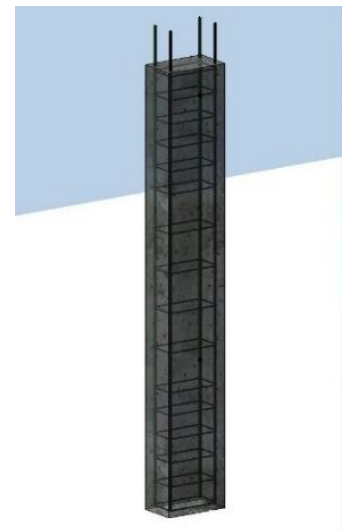

b)

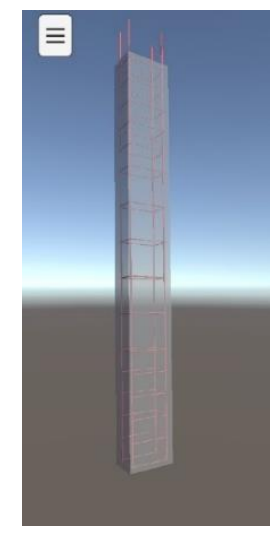

Fig. 1. Object representation: a) BIM software, b) Developed application

One of the issues encountered by the authors while preparing the exported models for further use was the location of a pivot point. By default, the usual location of the point is the center of gravity of the model. It was necessary to manually correct the location of the point, which was done by using special program for machining three-dimensional models.

Another dissimilarity was related to the texturing of objects. The textures from the BIM software were not exported through the *.3ds format, however, the transparency parameters and colors of elements were preserved. The main purpose was to represent reinforced 
concrete elements in a way allowing their optical verification, so the search for the solving this issue was omitted.

\section{Functionality of Developed Application}

The application was performed using $\mathrm{C} \#$ programming language and was compiled for mobile devices with Android operating system. Moreover, it was compiled basing on Android 4.0 that allows to use the application on older mobile devices as well.

The developed application serves to extend typical documentation with additional functionality, whilst not eliminating 2D Documentation, but rather using functionality as a supplement to 2D Documentation. Basically, it was agreed that the application should have the following features:

- Representation of reinforced concrete elements on the screen of a mobile device as a three-dimensional object in VR mode;

- Representation of reinforced concrete elements on the screen of a mobile device as a three-dimensional object in AR mode using 2D documentation as markers;

- The ability to add a comment, mark the related place and send this information to the corresponding person via e-mail;

- Attaching additional information about elements that were generated using the BIM software.

The mobile application was developed mainly for on-site purposes, allowing contractors quick verification of objects and direct communication with individual designers. The application`s functionality offers similar features as BIM-compliant browsers [10]. In case of doubts related to the documentation, contractor has the possibility to mark and comment related issue and send this information to the designer with the link or screenshot. Additionally, it was agreed that for this kind of projects, it would be appropriate to add reinforcement legend to corresponding objects. Figure 2a shows the screenshot from the developed application describing adding of comments and Figure $2 b$ shows the screenshot with an exemplary reinforcement legend.

a)

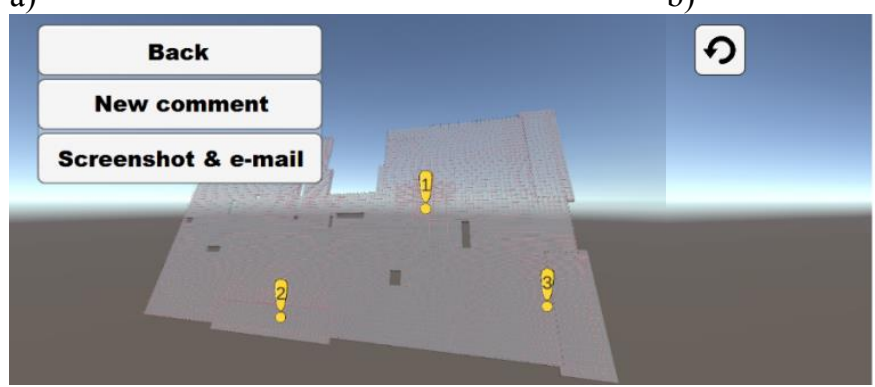

b)

Fig. 2. Screenshots from developed application: a) Adding comments; b) Exemplary legend

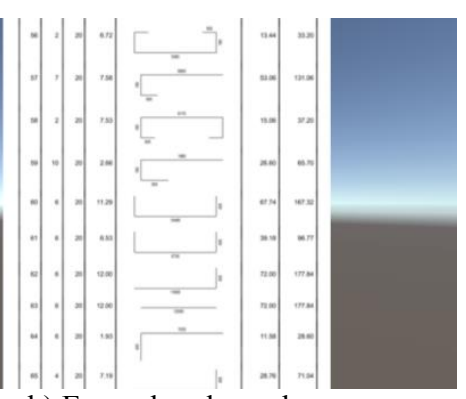

The effect of augmented reality additionally gives the opportunity to refer a threedimensional model to a reinforcement drawing [11], which can lead to the improving of the effectiveness and preventing the risk of construction errors [12]. When using a mobile device, it is possible to scroll the item and verify every region of component. The AR view of 3D model of the slab with top reinforcement is shown in the Figure 3. The model was set and oriented in space basing on 2D documentation that was used as AR marker. 


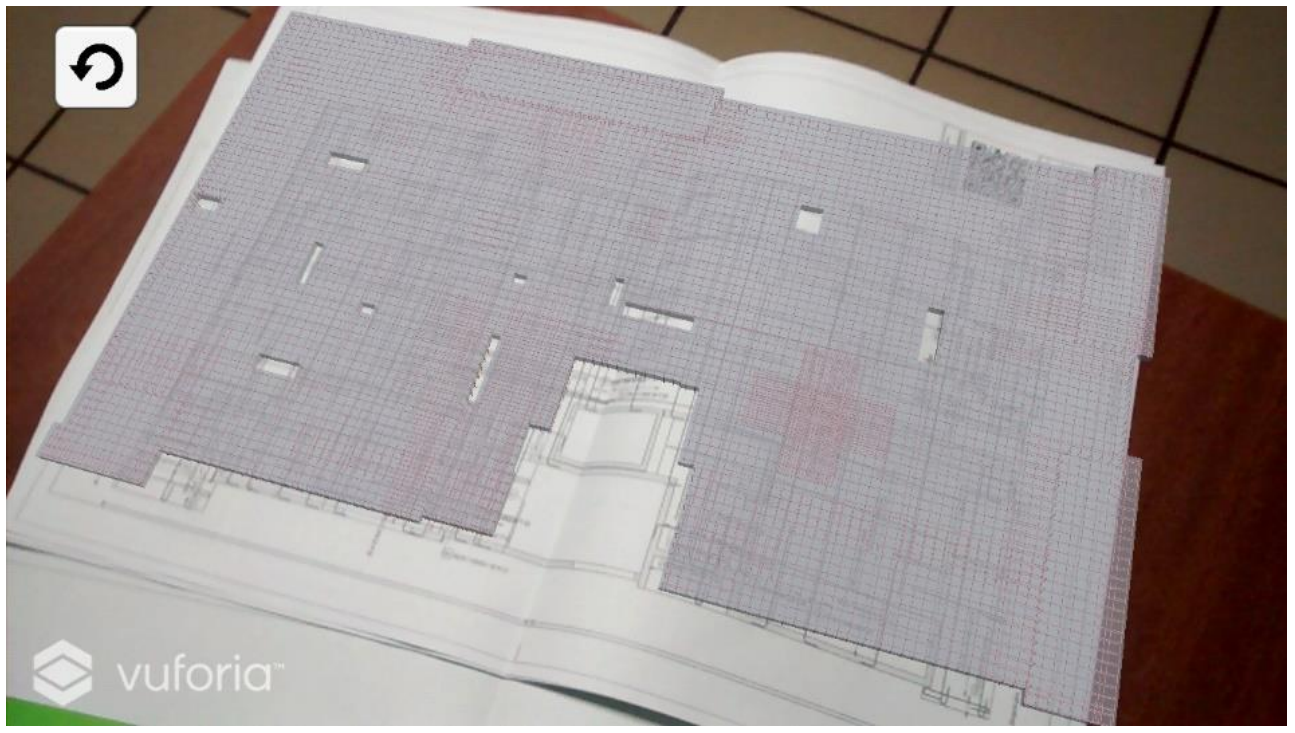

Fig. 3. Screenshot from developed application - AR mode for the slab with top reinforcement layer

\subsection{Remarks Regarding Correct Operation of Developed Application}

Some issues which can prevent to limitation of the use of the application were found. The essential necessities to reproduce object in AR mode are as following: illumination conditions, camera parameters and markers that contain sufficient number of characteristic points $[13,14]$.

The environment of the place, where the user wants to use the application to display the object in AR mode must be illuminated in such a way that the camera lens is able to recognize the marker of augmented reality. The problem with illumination may arise when using the application outside the building, where too bright light can be used to scan the image by the camera. A related point to consider is that the parameter regarding proper lighting depends on a large extent on the brightness of the lens in a given mobile device, so that the effect of AR may be unavailable in some conditions for a certain group of phones or tablets.

Definitely more requirements are put on markers that initiate the AR view. The quality of marker tracking deteriorates when they are bent. This condition is difficult to meet for ordinary drawings, which are usually submitted by A4 format for documentation purposes. Additionally, the markers of augmented reality must have a lack of gloss in order to limit reflections on their surface.

The Vuforia environment algorithm responsible for the generation of characteristic points, creates the number of characteristic points that are characterized by high contrast, many sharp edges and no repeating patterns. The first two requirements are met by typical technical drawings, but the last criterion deprives some of the drawings from being able to act as a marker of AR. On the example of one of such documents, the authors showed how its modification can improve the quality of displaying objects in AR view. To illustrate this process, a reinforced concrete column drawing was chosen, which was prepared with BIM software.

The number of characteristic points of a column section was insufficient for the correct displaying of objects. The Vuforia algorithm cannot recognize the marker due to repetitive components of the drawing. So, it was decided to insert a Quick Response (QR) code into 
drawing. A marked improvement in the quality of the marker detection has been achieved. However, it was also assumed that for correct and quick displaying of objects, the 3D representation, partial schemas and the reinforcement legends will be added to the drawing. Figure 4 describes the final 2D technical drawing for the column, which contains sufficient number of characteristic points to be used as AR marker.

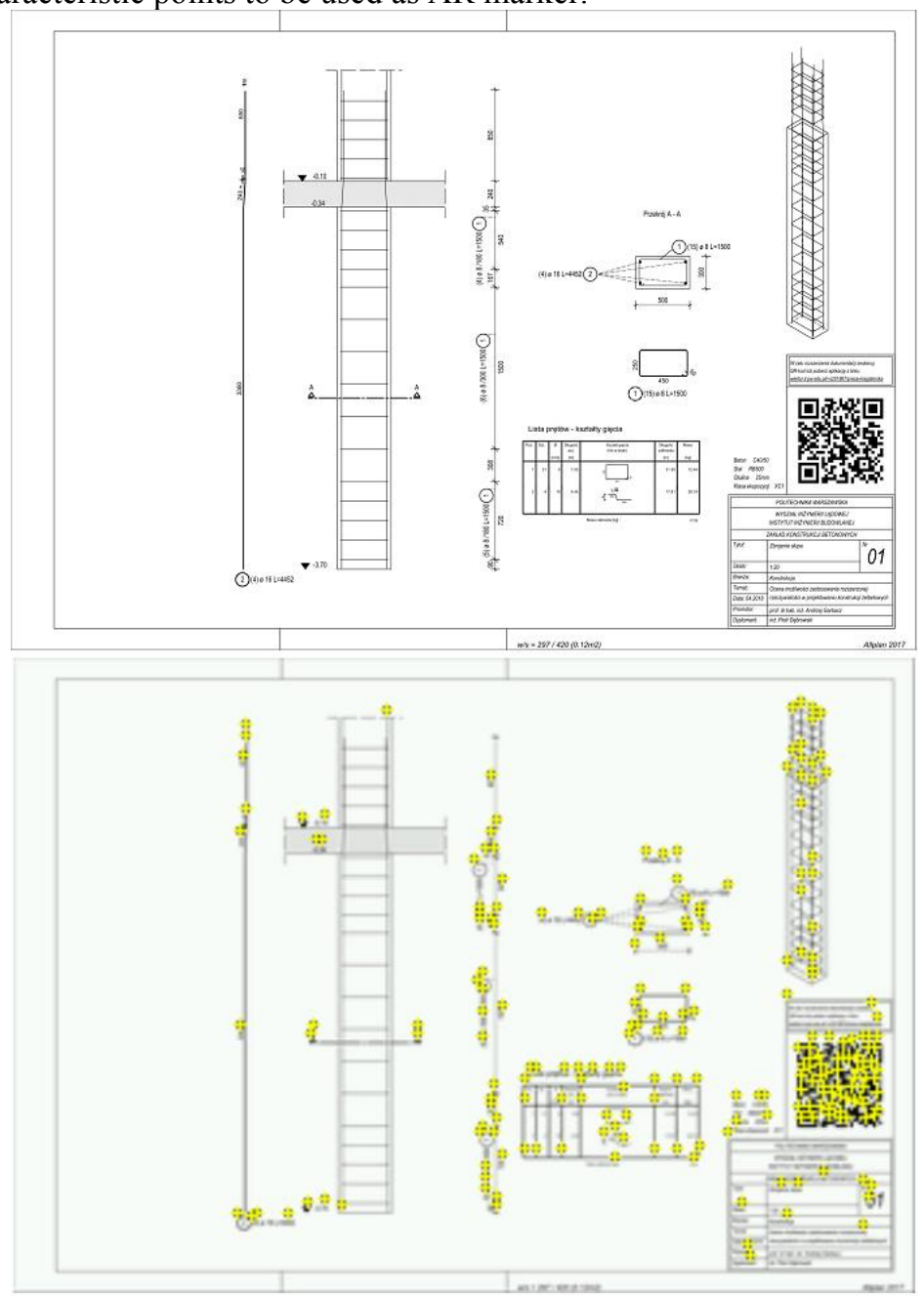

Fig. 4. Two-dimensional drawing with sufficient number of characteristic points allowing AR view

\section{Conclusions}

The functionality of the developed application is limited to displaying models and information about them. Nevertheless, the developed solution makes the verification of structural elements easier, as well as checking them before installation that can prevent the risk of making errors at the construction phase.

The preparation of BIM model, which will be used in developed application does not require a lot of work. Only the correct export and accurately made drawings are required. These activities slightly extend the standard design process using BIM-compliant software. Further work will be concentrated on extending technical abilities of the program and the 
use of artificial neural networks for allowing processing of big data bases and improving real-time collaboration on the project information [15].

Some issues which can limit the use of this application were discussed. The fundamental aspect to reproduce the objects in AR mode is an appropriate preparation of $2 \mathrm{D}$ documentation as markers for initiating AR view. This can be done with the proper illumination conditions and sufficient number of characteristic points. Additionally, the problem with older mobile devices can be omitted since it can be compiled for the devices based on older operating system.

\section{References}

1. M. Al Hattab, F. Hamzeh, Proceedings for the 21st Annual Conference of the International Group for Lean Construction, 80-90 (2013)

2. N. Kasim, IOP Conference Series: Materials Science and Engineering, 271, 012038 (2017)

3. M. Fischer, J. Kunz, Proceedings of JSCE, 763, 1-8 (2004)

4. B. A. Ellis, Government/Industry Forum by the Federal Facilities Council, 1-5 (2006)

5. J. M. Haymaker, C. Kam, M. Fischer, Construction Informatics Digital Library (2005)

6. P. Pardel, Studia Informatica, 82 (1), 35-41 (2009)

7. „Vuforia Developer Library,” [Online]. Available: https://library.vuforia.com/gettingstarted;

8. „Model file formats Unity Documentation,” [Online]. Available: https://docs.unity3d.com/Manual/3D-formats.html;

9. „Unity (game [Online]. Available: https://en.wikipedia.org/wiki/Unity_(game_engine);

10. M. Zaher, D. Greenwood, M. Marzouk, Construction Innovation, 18, 152-166 (2017)

11. M. Salamak, M. Januszka, T. Płaszczyk, Zeszyty Naukowo-Techniczne Stowarzyszenia Inżynierów i Techników Komunikacji w Krakowie, 3 (110), 137-151 (2016)

12. Oh-Seong Kwon, Chansik Park, Australasian Conference on Innovative Technologies in Construction, 93-97 (2012)

13. „Vuforia - Optimizing target detection and tracker stability,” [Online]. Available: https://library.vuforia.com/articles/Solution/Optimizing-Target-Detection-andTracking-Stability.html;

14. A.R. Singh, S. Vinayak, Venkata S. K. Delhi, Proceedings of the International Symposium on Automation and Robotics in Construction, 34, 1-8 (2017)

15. P. Knyziak, Proc. Eng. 91, 394-399 (2014) 